Network Working Group

Request for Comments: 2755

Category: Informational
A. Chiu

M. Eisler

B. Callaghan

Sun Microsystems

January 2000

\title{
Security Negotiation for WebNFS
}

Status of this Memo

This memo provides information for the Internet community. It does not specify an Internet standard of any kind. Distribution of this memo is unlimited.

Copyright Notice

Copyright (C) The Internet Society (2000). All Rights Reserved.

Abstract

This document describes a protocol for a WebNFS client [RFC2054] to negotiate the desired security mechanism with a WebNFS server [RFC2055] before the WebNFS client falls back to the MOUNT v3 protocol [RFC1813]. This document is provided so that people can write compatible implementations.

Table of Contents

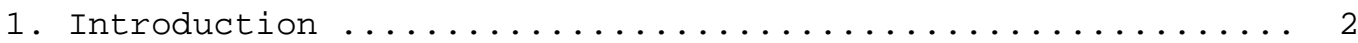

2. Security Negotiation Multi-component LOOKUP ........... 3

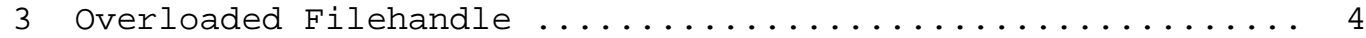

3.1 Overloaded NFS Version 2 Filehandle ................ 5

3.2 Overloaded NFS Version 3 Filehandle .............. 6

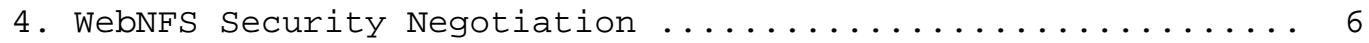

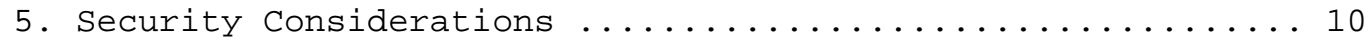

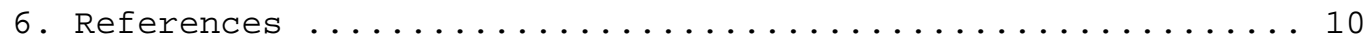

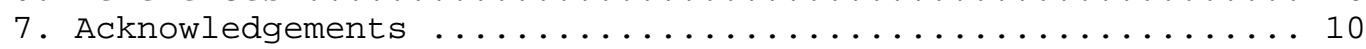

8. Authors' Addresses ........................ 11

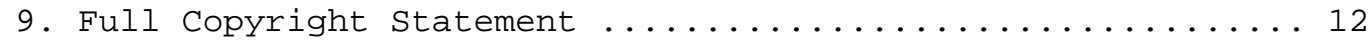




\section{Introduction}

The MOUNT protocol is used by an NFS client to obtain the necessary filehandle for data access. MOUNT versions 1 and 2 [RFC1094] return NFS version 2 filehandles, whereas MOUNT version 3 [RFC1813] returns NFS version 3 filehandles.

Among the existing versions of the MOUNT protocol, only the MOUNT v3 provides an RPC procedure (MOUNTPROC3_MNT) which facilitates security negotiation between an NFS V3 client and an NSF v3 server. When this RPC procedure succeeds (MNT3_OK) the server returns to the client an array of security mechanisms it supports for the specified pathname, in addition to an NFS v3 filehandle.

A security mechanism referred to in this document is a generalized security flavor which can be an RPC authentication flavor [RFC1831] or a security flavor referred to in the RPCSEC_GSS protocol [RFC2203]. A security mechanism is represented as a four-octet integer.

No RPC procedures are available for security negotiation in versions 1 or 2 of the MOUNT protocol.

The NFS mount command provides a "sec=" option for an NFS client to specify the desired security mechanism to use for NFS transactions. If this mount option is not specified, the default action is to use the default security mechanism over NFS v2 mounts, or to negotiate a security mechanism via the MOUNTPROC3_MNT procedure of MOUNT V3 and use it over NFS v3 mounts. In the latter, the client picks the first security mechanism in the array returned from the server that is also supported on the client.

As specified in RFC 2054, a WebNFS client first assumes that the server supports WebNFS and uses the publsc filehandle as the initial filehandle for data access, eliminating the need for the MOUNT protocol. The WebNFS client falls back to MOUNT if the server does not support WebNFS.

Since a WebNFS client does not use MOUNT initially, the MOUNTPROC3_MNT procedure of MOUNT v3 is not available for security negotiation until the WebNFS client falls back to MOUNT. A viable protocol needs to be devised for the WebNFS client to negotiate security mechanisms with the server in the absence of the MOUNTPROC3_MNT procedure. 
The WebNFS security negotiation protocol must meet the following requirements :

- Must work seamlessly with NFS v2 and v3, and the WebNFS protocols

- Must be backward compatible with servers that do not support this negotiation

- Minimum number of network turnarounds (latency)

This document describes the WebNFS security negotiation protocol developed by Sun Microsystems, Inc. Terminology and definitions from RFCs 2054 and 2055 are used in this document. The reader is expected to be familiar with them.

2. Security Negotiation Multi-component LOOKUP

The goal of the WebNFS security negotiation is to allow a WebNFS client to identify a security mechanism which is used by the WebNFS server to protect a specified path and is also supported by the client. The WebNFS client initiates the negotiation by sending the WebNFS server the path. The WebNFS server responds with the array of security mechanisms it uses to secure the specified path. From the array of security mechanisms the WebNFS client selects the first one that it also supports.

Without introducing a new WebNFS request, the WebNFS security negotiation is achieved by modifying the request and response of the existing multi-component LOOKUP (MCL) operation [RFC2055]. Note that the MCL operation is accomplished using the LOOKUP procedure (NFSPROC3_LOOKUP for NFS V3 and NFSPROC_LOOKUP for NFS V2). This and the next sections describe how the MCL request and response are modified to facilitate WebNFS security negotiation.

For ease of reference, the modified MCL request is henceforth referred to as SNEGO-MCL (security negotiation multi-component LOOKUP) request.

A multi-component LOOKUP request [RFC2055] is composed of a public filehandle and a multi-component path:

For Canonical Path:

LOOKUP $\mathrm{FH}=0 \times 0, " / \mathrm{a} / \mathrm{b} / \mathrm{c} "$ 
For Native Path:

$$
\text { LOOKUP } \mathrm{FH}=0 \times 0,0 \times 80 \mathrm{a}: \mathrm{b}: \mathrm{c} "
$$

A multi-component path is either an ASCII string of slash separated components or a 0x80 character followed by a native path. Note that a multi-component LOOKUP implies the use of the public filehandle in the LOOKUP.

Similar to the MCL request, a SNEGO-MCL request consists of a public filehandle and a pathname. However, the pathname is uniquely composed, as described below, to distinguish it from other pathnames.

The pathname used in a SNEGO-MCL is the regular WebNFS multicomponent path prefixed with two octets. The first prefixed octet is the 0x81 non-ascii character, similar to the 0x80 non-ascii character for the native paths. This octet represents client's indication to negotiate security mechanisms. It is followed by the security index octet which stores the current value of the index into the array of security mechanisms to be returned from the server. The security index always starts with one and gets incremented as negotiation continues. It is then followed by the pathname, either an ASCII string of slash separated canonical components or $0 \times 80$ and a native path.

A security negotiation multi-component LOOKUP request looks like this:

For Canonical Path:

LOOKUP $\mathrm{FH}=0 \times 0,0 \times 81<$ sec-index> "/a/b/c"

For Native Path:

LOOKUP FH=0x0, 0x81<sec-index> 0x80 "a:b:c"

In the next section we will see how the MCL response is modified for WebNFS security negotiation.

3. Overloaded Filehandle

As described in RFC2054, if a multi-component LOOKUP request succeeds, the server responds with a valid filehandle:

LOOKUP $\mathrm{FH}=\mathrm{O} \times \mathrm{O}, \mathrm{"} \mathrm{a} / \mathrm{b} / \mathrm{C} "$
$--------->$
$<-----------$

$\mathrm{FH}=0 \times 3$ 
NFS filehandles are used to uniquely identify a particular file or directory on the server and are opaque to the client. The client neither examines a filehandle nor has any knowledge of its contents. Thus, filehandles make an ideal repository for the server to return the array of security mechanisms to the client in response to a SNEGO-MCL request.

To a successful SNEGO-MCL request the server responds, in place of the filehandle, with an array of integers that represents the valid security mechanisms the client must use to access the given path. A length field is introduced to store the size (in octets) of the array of integers.

As the filehandles are limited in size (32 octets for NFS v2 and up to 64 octets for NFS V3), it can happen that there are more security mechanisms than the filehandles can accommodate. To circumvent this problem, a one-octet status field is introduced which indicates whether there are more security mechanisms ( 1 means yes, 0 means no) that require the client to perform another SNEGO-MCL to get them.

To summarize, the response to a SNEGO-MCL request contains, in place of the filehandle, the length field, the status field, and the array of security mechanisms:

FH: length, status, $\{$ sec_1 sec_2 ... sec_n $\}$

The next two sub-sections describe how NFS v2 and v3 filehandles are "overloaded" to carry the length and status fields and the array of security mechanisms.

\subsection{Overloaded NFS Version 2 Filehandle}

A regular NFS v2 filehandle is defined in RFC1094 as an opaque value occupying 32 octets:

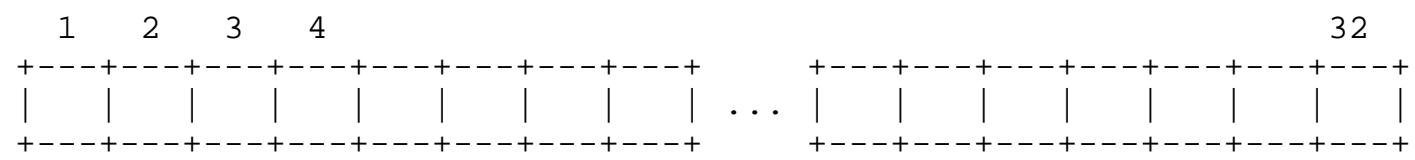

An overloaded NFS v2 filehandle looks like this:

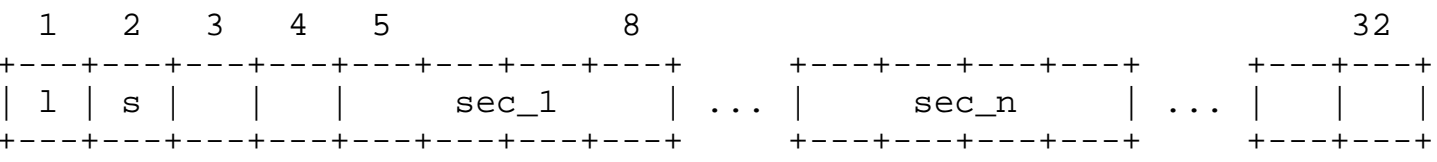


Note that the first four octets of an overloaded NFS v2 filehandle contain the length octet, the status octet, and two padded octets to make them XDR four-octet aligned. The length octet $1=4$ * $n$, where $\mathrm{n}$ is the number of security mechanisms sent in the current overloaded filehandle. Apparently, an overloaded NFS v2 filehandle can carry up to seven security mechanisms.

\subsection{Overloaded NFS Version 3 Filehandle}

A regular NFS V3 filehandle is defined in RFC1813 as a variable length opaque value occupying up to 64 octets. The length of the filehandle is indicated by an integer value contained in a four octet value which describes the number of valid octets that follow:
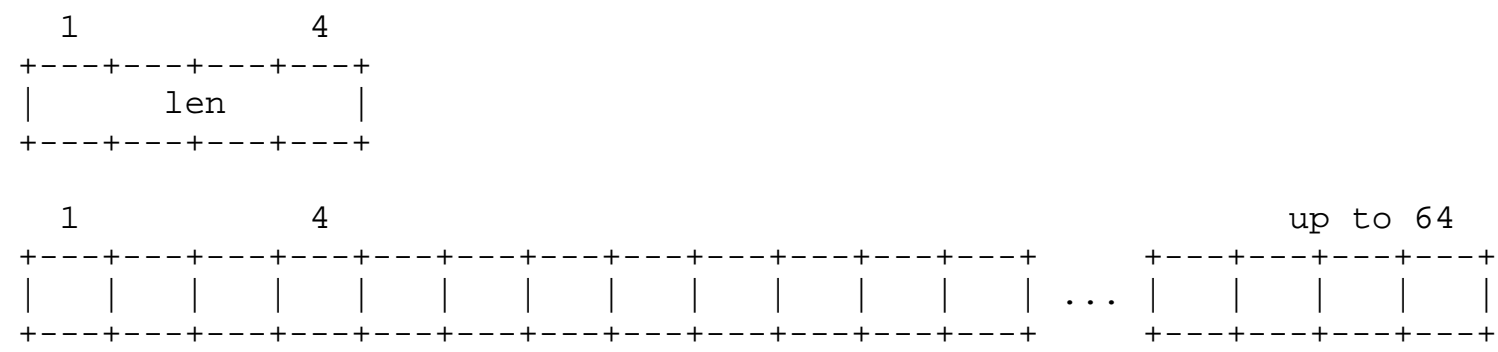

An overloaded NFS v3 filehandle looks like the following:
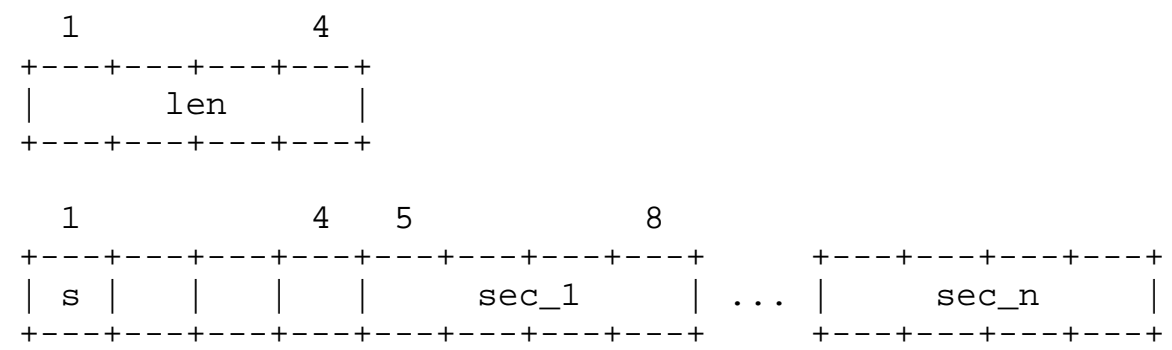

Here, len $=4 *(n+1)$. Again, $n$ is the number of security mechanisms contained in the current overloaded filehandle. Three octets are padded after the status octet to meet the XDR four-octet alignment requirement. An overloaded NFS v3 filehandle can carry up to fifteen security mechanisms.

4. WebNFS Security Negotiation

With the SNEGO-MCL request and the overloaded NFS v2 and v3 filehandles defined above, the following diagram depicts the WebNFS security negotiation protocol: 


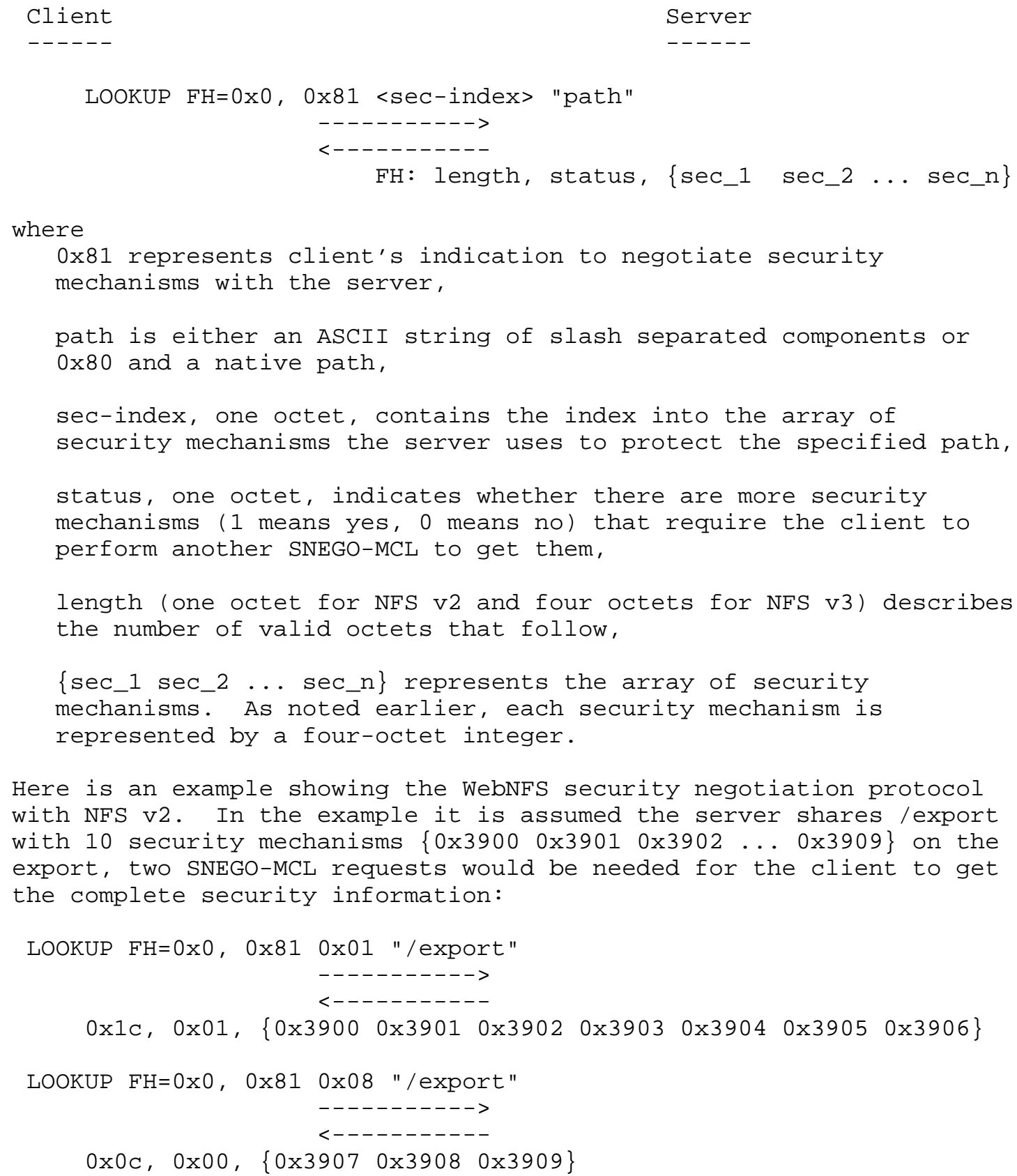


The order of the security mechanisms returned in an overloaded filehandle implies preferences, i.e., one is more recommended than those following it. The ordering is the same as that returned by the MOUNT v3 protocol.

The following shows a typical scenario which illustrates how the WebNFS security negotiation is accomplished in the course of accessing publicly shared filesystems.

Normally, a WebNFS client first makes a regular multi-component LOOKUP request using the public filehandle to obtain the filehandle for the specified path. Since the WebNFS client does not have any prior knowledge as to how the path is protected by the server the default security mechanism is used in this first multi-component LookUP. If the default security mechanism does not meet server's requirements, the server replies with the AUTH_TOOWEAK RPC authentication error, indicating that the default security mechanism is not valid and the WebNFS client needs to use a stronger one.

Upon receiving the AUTH_TOOWEAK error, to find out what security mechanisms are required to access the specified path the WebNFS client sends a SNEGO-qMCL request, using the default security mechanism.

If the SNEGO-MCL request succeeds the server responds with the filehandle overloaded with the array of security mechanisms required for the specified path. If the server does not support WebNFS security negotiation, the SNEGO-MCL request fails with NFSERR_IO for NFS v2 or NFS3ERR_IO for NFS v3 [RFC2055].

Depending on the size of the array of security mechanisms, the WebNFS client may have to make more SNEGO-MCL requests to get the complete array.

For successful SNEGO-MCL requests, the WebNFS client retrieves the array of security mechanisms from the overloaded filehandle, selects an appropriate one, and issues a regular multi-component LookUP using the selected security mechanism to acquire the filehandle.

All subsequent NFS requests are then made using the selected security mechanism and the filehandle.

The following depicts the scenario outlined above. It is assumed that the server shares /export/home as follows:

$$
\text { share }-0 \text { sec=sec_1:sec_2:sec_3, public/export/home }
$$


and AUTH_SYS is the client's default security mechanism and is not one of $\left\{\right.$ sec_l $_{1}$, sec_2, sec_3 3 .

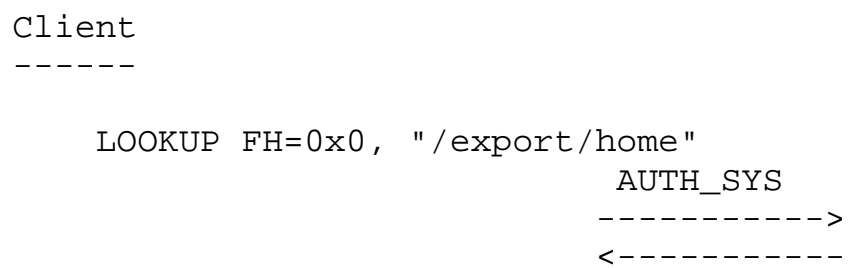




\section{Security Considerations}

The reader may note that no mandatory security mechanisms are specified in the protocol that the client must use in making SNEGOMCL requests. Normally, the client uses the default security mechanism configured on his system in the first SNEGO-MCL request. If the default security mechanism is not valid the server replies with the AUTH_TOOWEAK error. In this case the server does not return the array of security mechanisms to the client. The client can then make another SNEGO-MCL request using a stronger security mechanism. This continues until the client hits a valid one or has exhausted all the supported security mechanisms.

6. References

[RFC1094] Sun Microsystems, Inc., "NFS: Network File system Protocol Specification", RFC 1094, March 1989. http://www.ietf.org/rfc/rfc1094.txt

[RFC1813] Callaghan, B., Pawlowski, B. and P. Staubach, "NFS Version 3 Protocol Specification", RFC 1813, June 1995. http://www.ietf.org/rfc/rfc1813.txt

[RFC2054] Callaghan, B., "WebNFS Client Specification", RFC 2054, October 1996. http://www.ietf.org/rfc/rfc2054.txt

[RFC2055] Callaghan, B., "WebNFS Server Specification", RFC 2055, October 1996. http://www.ietf.org/rfc/rfc2055.txt

[RFC2203] Eisler, M., Chiu, A. and Ling, L., "RPCSEC_GSS Protocol Specification", RFC 2203, september 1997. http: //www.ietf.org/rfc/rfc2203.txt

7. Acknowledgements

This specification was extensively brainstormed and reviewed by the NFS group of Solaris Software Division. 
8. Authors' Addresses

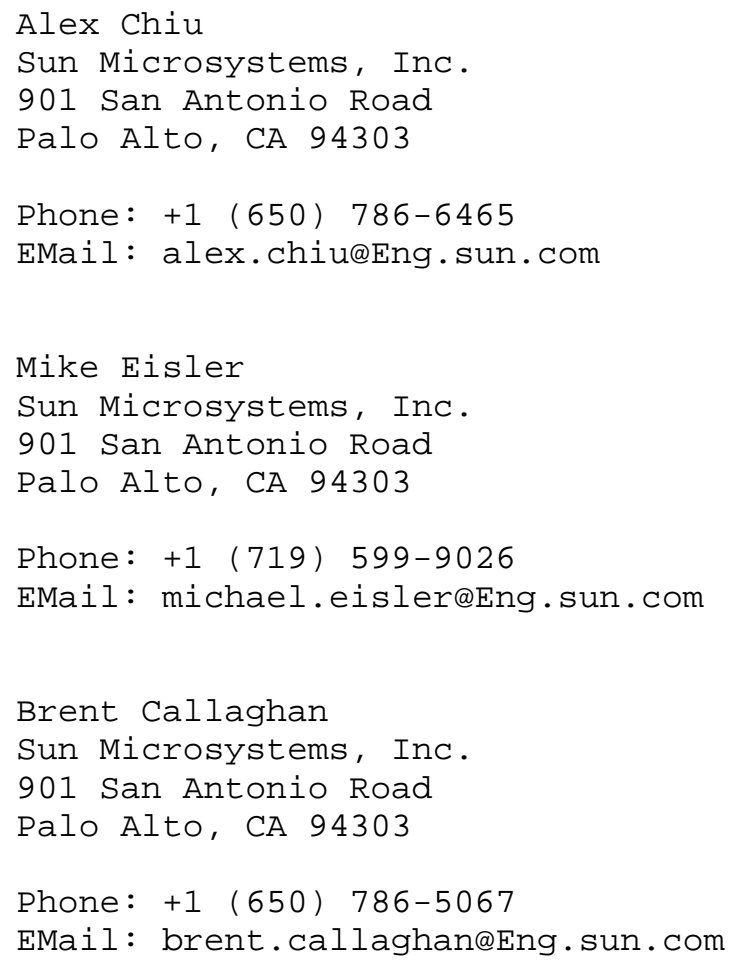


9. Full Copyright Statement

Copyright (C) The Internet Society (2000). All Rights Reserved.

This document and translations of it may be copied and furnished to others, and derivative works that comment on or otherwise explain it or assist in its implementation may be prepared, copied, published and distributed, in whole or in part, without restriction of any kind, provided that the above copyright notice and this paragraph are included on all such copies and derivative works. However, this document itself may not be modified in any way, such as by removing the copyright notice or references to the Internet Society or other Internet organizations, except as needed for the purpose of developing Internet standards in which case the procedures for copyrights defined in the Internet Standards process must be followed, or as required to translate it into languages other than English.

The limited permissions granted above are perpetual and will not be revoked by the Internet society or its successors or assigns.

This document and the information contained herein is provided on an "AS IS" basis and THE INTERNET SOCIETY AND THE INTERNET ENGINEERING TASK FORCE DISCLAIMS ALL WARRANTIES, EXPRESS OR IMPLIED, INCLUDING BUT NOT LIMITED TO ANY WARRANTY THAT THE USE OF THE INFORMATION HEREIN WILL NOT INFRINGE ANY RIGHTS OR ANY IMPLIED WARRANTIES OF MERCHANTABILITY OR FITNESS FOR A PARTICULAR PURPOSE.

Acknowledgement

Funding for the RFC Editor function is currently provided by the Internet Society. 